\title{
A Foe Incognito: Paraneoplastic Sacroilitis
}

\author{
Sumantro Mondal ${ }^{\mathrm{a}}$, Debanjali Sinha ${ }^{\mathrm{a}}$, Tony Ete ${ }^{\mathrm{b}, \mathrm{d}}$, Rudra Prosad Goswami ${ }^{\mathrm{a}}$, Jayati Bardhan ${ }^{\mathrm{c}}$, \\ Alakendu Ghosh ${ }^{\mathrm{a}}$
}

\begin{abstract}
Paraneoplastic rheumatological symptoms are not uncommon in clinical practice. They can predate the diagnosis of the neoplastic disease. Carcinomatous polyarthritis, vasculitis, and inflammatory myopathy are commonly encountered paraneoplastic rheumatological diseases. Paraneoplastic sacroiliitis is rather uncommon. We herein report a case of paraneoplastic sacroiliitis due to acute myeloid leukemia in a young male.
\end{abstract}

Keywords: Sacroilitis; Spondyloarthropathy; Paraneoplastic syndrome

\section{Introduction}

Paraneoplastic involvement of musculoskeletal tissues is close mimicker of various rheumatological diseases and often creates diagnostic challenge to the clinician. Rheumatological symptoms can appear before the diagnosis of original neoplasia. Carcinomatous polyarthritis, vasculitis, and inflammatory myopathy are considered as common paraneoplastic rheumatological diseases. Paraneoplastic involvement of sacroiliac joints is rather uncommon. In daily practice, low back pain combined with peripheral arthritis and MRI documented sacroiliitis are very much suggestive of diagnosing spondyloarthritis (SpA), especially in a young male. However, a clinician should also be aware of close mimickers and other differentials. From the imaging viewpoint, infective and neoplastic etiology of sacroiliitis should also be considered among the differentials. We herein report a case of paraneoplastic sacro-

Manuscript accepted for publication May 25, 2016

aDepartment of Rheumatology, Institute of Post Graduate Medical Education and Research, 244 A J C Bose Road, Kolkata, India

${ }^{b}$ Department of Medicine, Institute of Post Graduate Medical Education and Research, 244 A J C Bose Road, Kolkata, India

'Department of Radiology, Bankura Sammilani Medical College and Hospital, Bankura, India

${ }^{\mathrm{d} C}$ Corresponding Author: Tony Ete, Department of Medicine, Institute of Post Graduate Medical Education and Research, 244 A J C Bose Road, Kolkata, India. Email: tetepete@gmail.com

doi: http://dx.doi.org/10.14740/jmc2540e iliitis due to acute myeloid leukemia in a young male along with peripheral arthritis mimicking SpA.

\section{Case Report}

A 27-year-old male patient was admitted with history of low back pain associated with asymmetric large joint arthritis involving his ankles and knees for last 1 year. His back pain was insidious in onset, progressively severe in nature ( 8 out of 10 in a visual analogue scale), associated with morning stiffness, persisted throughout the day but aggravated at night with frequent awakening due to pain. He required three to four NSAIDs daily with inadequate response. He had no history of uveitis, dactylitis, enthesitis, psorariform skin rash, diarrhea or similar illness in his family. He was hospitalized 3 months for his back pain and detected to have anemia for which he was transfused with four units of whole blood. There was significant anorexia and weight loss and he was almost bed ridden for his illness. He had no history of any previous mucosal bleeding or frequent infection. Examination revealed moderate pallor, tachycardia, hepatomegaly (hepatic span: $16 \mathrm{~cm}$ ) and a grade III systolic murmur at pulmonary area. Musculoskeletal examination showed tenderness of bilateral sacroiliac joints along with synovitis of bilateral knee and right ankle joints. Tenderness was present over costochondral junctions and adjacent sternum. No skin or nail lesion was found. Ophthalmological examination did not reveal any evidence of uveitis. Blood investigation reports showed anemia ( $\mathrm{Hb}: 7.2 \mathrm{~g} / \mathrm{dL}$ ), total leukocyte count (TLC) of $11,200 / \mathrm{mm}^{3}$ (neutrophil: $52 \%$, lymphocyte: $43 \%$, eosinophil: $4 \%$ ), platelets of $1.1 \mathrm{lakhs} / \mathrm{mm}^{3}$ and ESR of $55 \mathrm{~mm} \mathrm{Hg}$ first hour. Peripheral blood smear showed normochromic, normocytic RBCs without any abnormal WBCs. All other investigations including, ferrokinetics, ICTC, stool occult blood test and upper GI endoscopy were normal. He was negative for HLA-B 27 and X-ray of sacroiliac joints was normal. Color Doppler ultrasound of his sacroiliac joints (Fig. 1A) revealed increased vascularity with low resistance flow pattern suggestive of sacroiliac joint inflammation, later on confirmed by MRI documentation of bilateral sacroiliitis (Fig. 1B). He was treated with adequate doses of indomethacin oral formulation without any satisfactory response. His subsequent blood investigation showed bicytopenia $(\mathrm{Hb}: 6.4 \mathrm{~g} / \mathrm{dL}$, platelets: $40,000 / \mathrm{mm}^{3}$ ) along with leucocytosis (TLC: $15,500 /$ $\mathrm{mm}^{3}, \mathrm{~N} 32 \mathrm{L67}$ ). The presence of bicytopenia urged us to do a bone marrow aspiration study. Bone marrow aspiration study 



Figure 1. (A) Color Doppler ultrasound of his sacroiliac joint showing increased vascularity of the joint. (B) MRI of sacroiliac joint showing bilateral sacroiliitis.

showed acute myeloid leukemia (Fig. 2). He was finally diagnosed as acute myeloid leukemia with paraneoplastic sacroiliitis and arthritis and was referred to department of hematology for further management.

\section{Discussion}

The clinical scenario of this young male was very much suggestive for a diagnosis of axial SpA. He fulfilled the ASAS criteria for classification of axial SpA. However, SpA could not explain some unusual clinical features present in this patient. Apparently his back pain fulfilled the criteria proposed by ASAS expert committee but with little atypicality. There was no improvement of pain with activities, persistent pain throughout whole day though more severe in night hours and no relief of night pain upon getting up. Unsatisfactory response to multiple doses of NSAIDs suggested unusual for

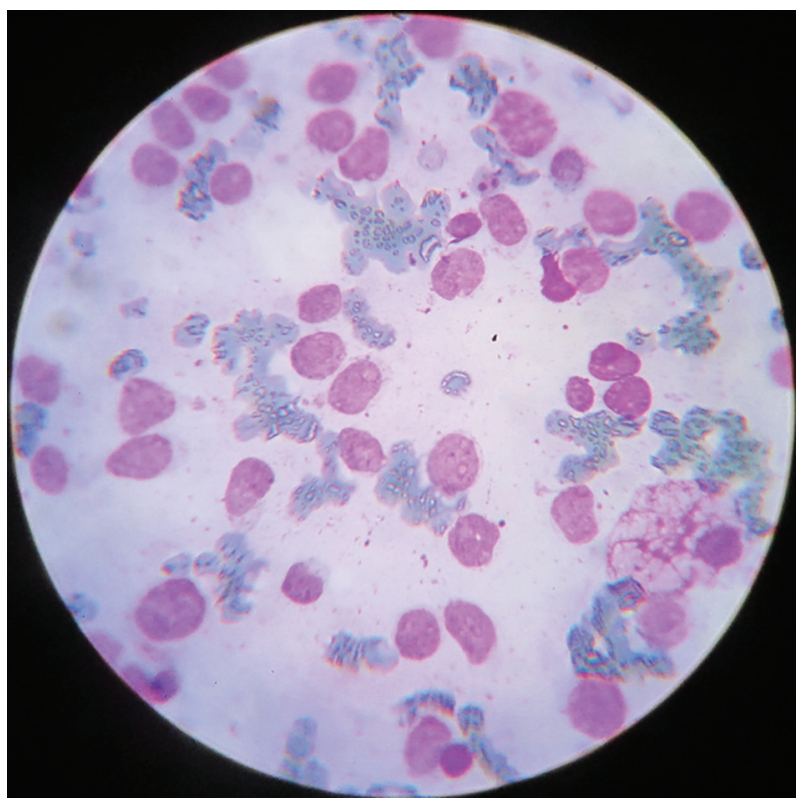

Figure 2. Bone marrow aspiration study showing abnormal myeloid cells.
SpA.

Presence of severe anemia requiring multiple unit blood transfusion also could not be explained by SpA. Failure to detect any occult GI bleeds and normal endoscopic findings almost ruled out iron deficiency anemia due to overuse of NSAIDs, which was further supported by his peripheral blood smear picture and ferrokinetics parameters. Subsequent documentation of thrombocytopenia in addition to progressive anemia led us to search for hematologic malignancy.

Rheumatic syndromes can develop as a manifestation of a paraneoplastic process. These are not due to direct invasion of the tumor or metastatic process. Carcinomatous polyarthritis, vasculitis, inflammatory myopathy, and Raynaud's phenomenon are the common paraneoplastic rheumatic symptoms [1]. Detailed pathogenesis for paraneoplastic arthritis is yet to be established. Aberrant immunoregulation or modulation of immunogenetics may be a predisposing factor [2]. Recent studies revealed the roles of mediators such as vascular endothelial growth factor and fibroblast growth factor 23 [3]. In a study in 139 patients with adult acute leukemia, 5.8\% were found to present with rheumatic manifestations [4]. Rheumatic symptoms can predate the diagnosis of the original neoplastic disorder. In patients with AML, rheumatic syndromes may precede diagnosis by an average of 3 months to a maximum of 2 years [5]. In a recent study with 10 patients malignancies were diagnosed after rheumatic symptoms in all patients. Diagnosis of haematological malignancies is more delayed than the solid tumors [6]. Paraneoplastic sacroiliitis is a rarely described spectrum with only few reported cases till date. Sacroiliitis and seronegative polyarthritis have been reported with B- and T-cell lymphomas [7]. Previously Cohen et al documented rapidly progressive sacroiliitis in a 61-yearold woman with diffuse well differentiated lymphocytic lymphoma. In this patient, disease onset with pain and stiffness in the lower back and the rapidity of symptom progression was much similar to our patient [8]. In a case report by Hoshino et al, sacroiliitis was the initial manifestation of AML in a 28 -year-old female. It was also suggested that autoimmune sacroiliitis was a paraneoplastic phenomenon of AML [9]. Similarly, Bahar Artim Esen et al reported biphenotypic leukemia in a 40-year-old woman with a presentation mimicking $\mathrm{SpA}$. There was also striking unresponsiveness to conventional anti-rheumatic treatment [10]. Distinctive features of 
paraneoplastic arthritis include severe pain disproportionate to physical examination, rapid progression, poor response to NSAIDs and abnormal radiologic findings like lytic bone lesions and early osteopenia.

Over the last decade, MRI became an invaluable tool for detecting sacroiliitis in early non-radiographic stage. However, it is also important to differentiate sacroiliitis from its mimickers like infection, insufficiency fracture and neoplastic infiltration. Inflammation unrestricted to anatomical borders, abnormal enhancement of soft tissues other than synovium, capsule or enthuses or presence of lytic bone lesion in visualized pelvic bone or spine should be interpreted with caution.

\section{Conclusions}

Paraneoplastic rheumatic symptoms may mask the original neoplastic disease.

SpA-like presentation is not so rare for hematological malignancies and atypical clinical spectrum needs careful evaluation for exclusion of malignancy.

MRI of sacroiliac joints needs cautious interpretation for exclusion of sacroiliitis mimickers.

\section{Source of Support}

None.

\section{Conflicts of Interest}

None.

\section{References}

1. Swierkot J, Lewandowicz-Uszynska A, Bogunia-Kubik K. [Paraneoplastic syndromes and rheumatic diseases]. Postepy Hig Med Dosw (Online). 2014;68:944-954.

2. Caldwell DS. Musculoskeletal syndrome associated with malignancy. In: Kelley WN, Harris ED Jr, Ruddy S, Sledge CB, eds. Textbook of Rheumatology. Saunders, Philadelphia. 1989. p. 1674-1689.

3. Manger B, Schett G. Paraneoplastic syndromes in rheumatology. Nat Rev Rheumatol. 2014;10(11):662-670.

4. Gur H, Koren V, Ehrenfeld M, Ben-Bassat I, Sidi Y. Rheumatic manifestations preceding adult acute leukemia: characteristics and implication in course and prognosis. Acta Haematol. 1999;101(1):1-6.

5. Naschitz JE, Rosner I, Rozenbaum M, Zuckerman E, Yeshurun D. Rheumatic syndromes: clues to occult neoplasia. Semin Arthritis Rheum. 1999;29(1):43-55.

6. Yamashita H, Ueda Y, Ozaki T, Tsuchiya H, Takahashi Y, Kaneko H, Kano T, et al. Characteristics of 10 patients with paraneoplastic rheumatologic musculoskeletal manifestations. Mod Rheumatol. 2014;24(3):492-498.

7. Conaghan PG, Brooks PM. Rheumatic manifestations of malignancy. Curr Opin Rheumatol. 1994;6(1):105-110.

8. Cohen MR, Carrera GE, Lundberg J. Rapidly progressive sacroiliitis in a patient with lymphocytic lymphoma. Ann Rheum Dis. 1993;52(3):239-240.

9. Hoshino T, Matsushima T, Saitoh Y, Yamane A, Takizawa M, Irisawa H, Saitoh T, et al. Sacroiliitis as an initial manifestation of acute myelogenous leukemia. Int J Hematol. 2006;84(5):421-424.

10. Bahar Artim Esen, Leyla Ozer, Sevil Kamali, Murat Inanc. Eur J Gen Med. 2011;8(2):151-153. 